\title{
SOUTHERN AFRICAN CASE STUDIES OF VARIATIONS IN INDICATOR MINERAL CHARACTERISTICS WITH DISTANCE FROM KIMBERLITE SOURCE
}

\author{
Brett van Coller $^{1}$, Peter Hildebrand ${ }^{1}$, Dale Verran ${ }^{1}$, Florence Barnes ${ }^{1}$, Tom Nowicki ${ }^{2}$, Mike \\ Baumgartner ${ }^{1}$, Larry Ott ${ }^{3}$, John Gurney ${ }^{1}$ \\ ${ }^{1}$ Mineral Services South Africa Pty Ltd; ${ }^{2}$ Mineral Services Canada Inc, Vancouver, ${ }^{3}$ Motapa Diamonds Inc.
}

\section{INTRODUCTION}

An investigation into the variation of kimberlitic indicator mineral (KIM) abundance, type, grain size, and surface textures with distance from source has been carried out over three known kimberlite localities in southern Africa. All three localities are located within arid Kalahari environment. The geology of each is summarized in Table 1. Locality 1 and 2 are subject to present day aeolian transport mechanisms; Locality 3 experiences both aeolian and fluvial sheet wash type, sediment transport. Vegetation cover at Locality 1 and 2 consists primarily of stunted mopani scrub interspersed with grass. Vegetation cover is markedly thinner over the Locality 3 area, and is in some places is entirely absent.

Sampling programs carried out over each locality had the following objectives:

Locality 1: Determine the size, nature and extent of KIM halo developed over a kimberlite in the Kalahari environment, together with the concentration, surface texture and size fraction proportions of the KIMs that comprise these halos. Determine the effect of varying heavy mineral trap site "quality" during routine exploration.

Locality 2: Test the consistency of KIM abundances and dispersion haloes over kimberlites in this terrain and investigate the relationship between deflation and loam samples collected at the same site at varying distances from kimberlite pipes.

Locality 3: Determine the effect of aeolian and fluvial transport processes on KIM distribution together with their effect on KIM grain surface morphology.

Results are discussed in terms of practical implications for kimberlite exploration programs. In particular, the usefulness and limitations of the methods and data are considered.

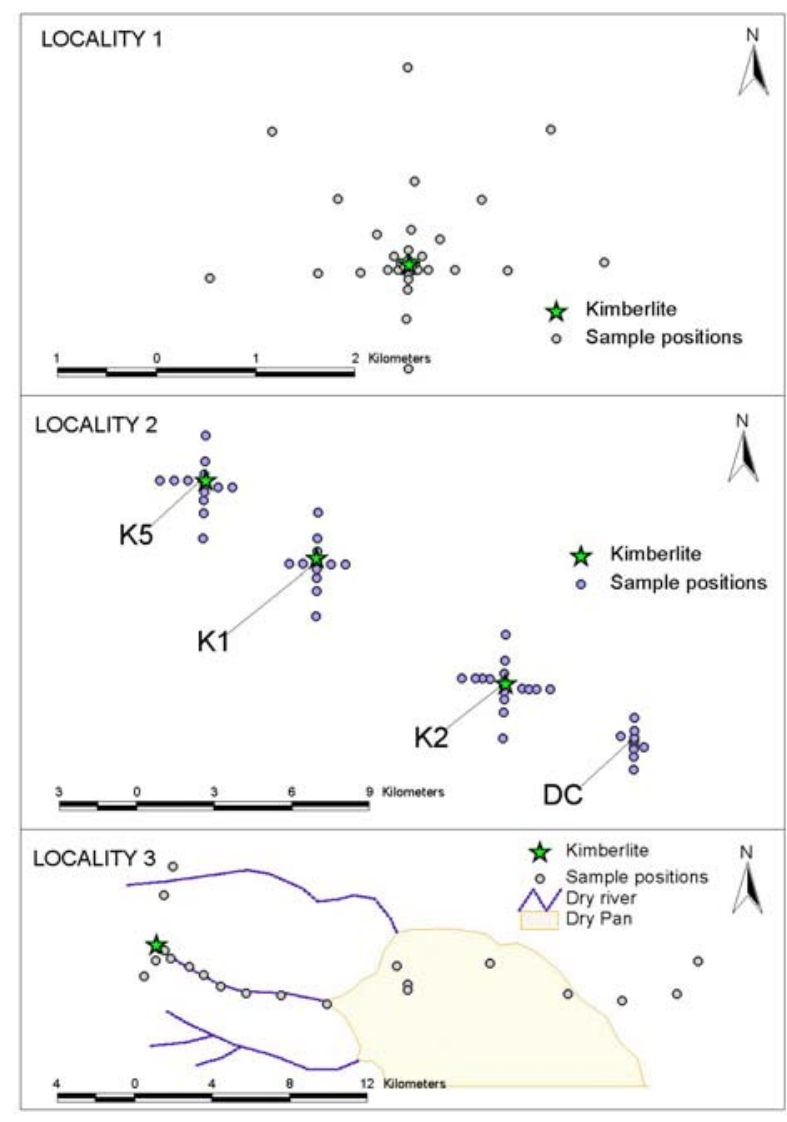

Figure 1: Location of sample sites at locality 1, 2 and 3

\section{METHODS}

\section{SAMPLE COLLECTION}

Two different sampling techniques were employed, i.e. deflation sweep and loam. Deflation sweep samples comprised the collection of the top 2-3 cm of soil cover over an area of approximately $30 \mathrm{~m}^{2}$ at each site. The distribution of deflation sweep samples was not dependent on the presence of well developed deflation sites, and samples were collected from deflation surfaces within a few meters of the planned sample site. Therefore the deflation surfaces sampled 
Table 1: Summary details of the three localities evaluated in this study

\begin{tabular}{|c|c|c|c|c|}
\hline & Nature of Occurrence & ${ }^{*}$ Size $(\mathrm{m})$ & $\begin{array}{l}\text { Total Cover } \\
\text { thickness }(\mathrm{m})\end{array}$ & $\begin{array}{l}\text { Cover } \\
\text { Lithology }\end{array}$ \\
\hline Locality 1 & Single pipe within a cluster & 100 & 30 & $\begin{array}{l}\text { Kalahari sand, } \\
\text { sand stone, } \\
\text { limestone }\end{array}$ \\
\hline Locality 2 & $\begin{array}{l}\text { Four pipes and a kimberlite dyke } \\
\text { variable width }\end{array}$ & $\begin{array}{l}\text { Pipes range in } \\
\text { size from } 300 \\
\text { to } 60 \mathrm{~m} \text { and } \\
\text { dyke } 1-10 \mathrm{~m} \\
\text { wide }\end{array}$ & 50 & $\begin{array}{l}\text { Kalhari sand, } \\
\text { sandstone }\end{array}$ \\
\hline Locality 3 & $\begin{array}{l}\text { Isolated pipe extensively pipped } \\
\text { and located in zone of active } \\
\text { errosion. }\end{array}$ & 80 & $0-2$ & $\begin{array}{l}\text { Calcretized } \\
\text { horizon }\end{array}$ \\
\hline
\end{tabular}

* Size inferred from geophysical modelling

varied significantly among sample sites, and each site was allocated a trap site quality grading. Trap site quality is a reflection of the degree of deflation surface development at each site. Loam samples comprised of material collected from a pit dug to a depth of approximately $30 \mathrm{~cm}$, excluding the top $3 \mathrm{~cm}$ of deflation material which was first removed. Deflation samples were collected at all sites but loam samples were only collected in conjunction with deflation samples at selected sites. All samples were sieved on site to obtain an approximate final weight of either 5 or $10 \mathrm{~kg}$ of $-2 \mathrm{~mm},+0.425 \mathrm{~mm}$ sample material. The initial volume of material required to obtain the desired final sample weight varied significantly among sample sites. Detailed sample site information was recorded for each sample, including notes on trap site quality, vegetation cover, initial sample volume, soil texture, soil colour and geomorphology.

The distribution of sample sites at each locality is shown in Figure 1.

\section{SAMPLE PROCESSING}

All samples were processed using standard screening and dense media separation procedures currently employed by Mineral Services Diamond Laboratory for kimberlite exploration samples. The concentrates obtained were examined for KIMs (garnet, chromite, ilmenite and chrome diopside). In cases where the abundance of KIMs was high the concentrate was split and a representative number of grains extracted.
Surface textures of recovered KIMs were described in terms of grain angularity and the degree of preservation of "primary" surface textures (i.e. those relating to etching / alteration of the KIM grain within the kimberlite magma).

\section{ABUNDANCE AND RATIO CALCULATIONS}

Mineral abundance totals presented here are calculated for the number of $+0.425 \mathrm{~mm}$ grains picked and normalized to a screened sample weight of $5 \mathrm{~kg}$. Mineral type, size and surface texture ratios were determined for all grains picked, including grains in the $-0.425 \mathrm{~mm}$ fraction.

\section{RESULTS}

Results reported for localities 1 and 2 relate almost exclusively to ilmenite as few garnets or other KIMs were recovered. A full range of KIMs were recovered at Locality 3 although the majority of grains recovered were purple garnets.

\section{KIMBERLITE INDICATOR MINERAL HALOS}

\section{Abundance}

A well defined and very restricted halo was obtained over Locality 1, with normalized KIM abundance dropping off from an average of 150 over the pipe to a low of 8 grains $200 \mathrm{~m}$ from the pipe center. The KIM halo is not distorted due to paleo or prevailing wind 
transport direction. The total ilmenite abundance recovered at specific distances from the pipe are presented in Figure 2.

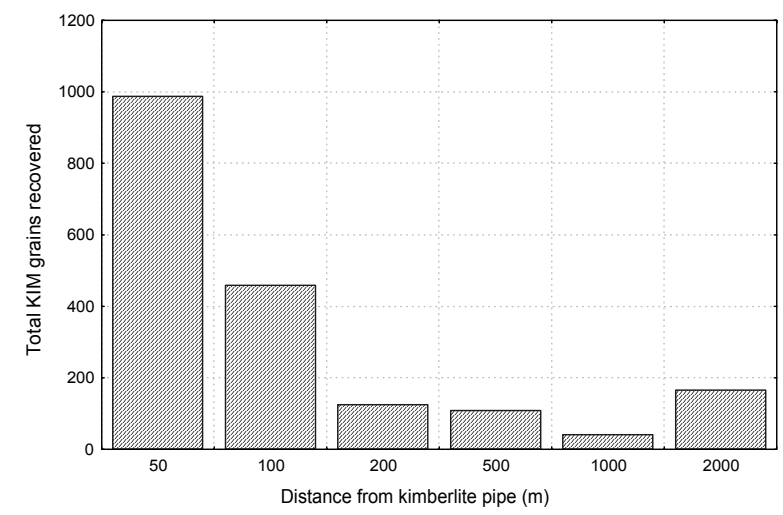

Figure 2: Total abundance of ilmenite grains recovered from deflation samples collected at varying distances from the Locality 1 kimberlite pipe.

All four kimberlites tested at Locality 2 show a similar drop off in KIM counts with distance from each pipe center (Figure 3). KIM abundances obtained from kimberlite K2 samples (Figure 3) are an order of magnitude larger than those obtained over the remaining 3 kimberlite bodies. This may relate to the KIM content of the individual kimberlite.

A definable drop-off in KIM counts with distance for deflation samples collected within the dry river channel at Locality 3 is observed (Figure 4).

\section{Grain size}

Results over Locality 1 indicate that no clear correlation exists between the percentage of different KIM grain size fractions (calculated for each sample) with distance from the pipe center. Large $+1 \mathrm{~mm}$ grains are however restricted to within $200 \mathrm{~m}$ from the pipe center.

Locality 3 shows a subtle decrease in the variation of KIM grain size with distance from the pipe. This may be attributed to a greater degree of sorting. It is also evident that KIM grain size fractions recovered at each site are a function of the energy regime at that particular site and not entirely a function of distance from kimberlite source in this particular terrain.

\section{Mineral type}

The percentage of KIM types was calculated for samples collected at each locality. No clear trends were observed although a significant number of chrome diopside grains were recovered at sample sites up to a distance of $13 \mathrm{~km}$ from the Locality 3 pipe.

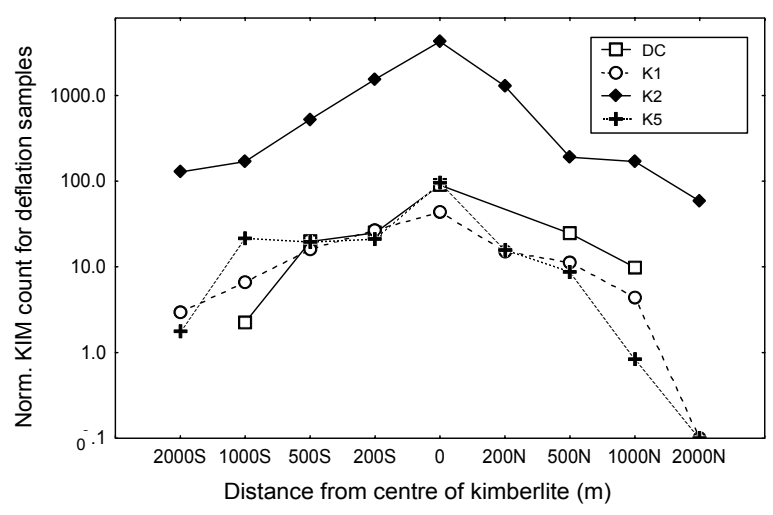

Figure 3: Decrease in abundance of KIMs with distance from all four kimberlite bodies at Locality 2

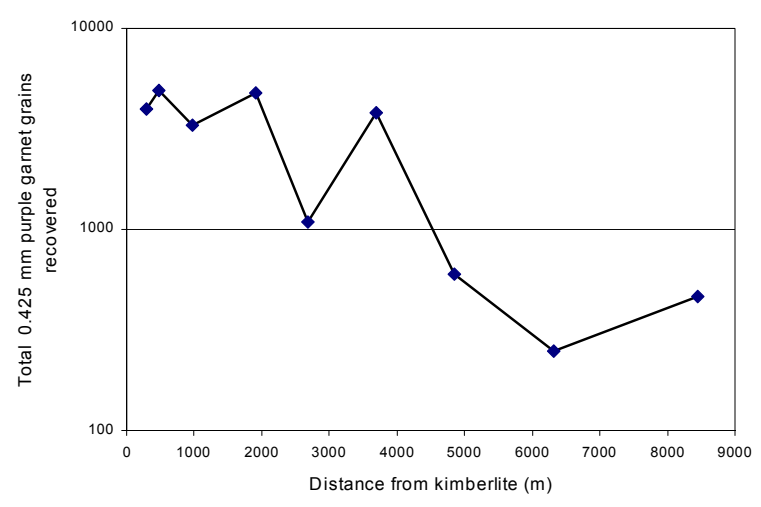

Figure 4: Decrease in abundance of $0.425 \mathrm{~mm}$ size fraction purple garnets recovered from within the dry river bed with increasing distance from kimberlite Locality 3.

\section{Surface textures}

A strong correlation between surface features of ilmenite grains and distance from pipe was observed at Locality 1. Perovskite mantled ilmenites (PMI) are constrained to within $500 \mathrm{~m}$ of the Locality 1 pipe center.

A decrease in the percentage of PMIs recovered relative to distance from each of the 4 Locality 2 kimberlite bodies is shown in Figure 5.

An increase in angularity of KIM grains with distance from the Locality 3 pipe is observed for deflation samples collected within the dry river channel (Figure 6). A possible explanation is mechanical breaking/shattering of grains as a result of flash flood events. Extensively abraided KIM grains were mostly 
observed outside the drainage features within aeolian sample/trap sites.

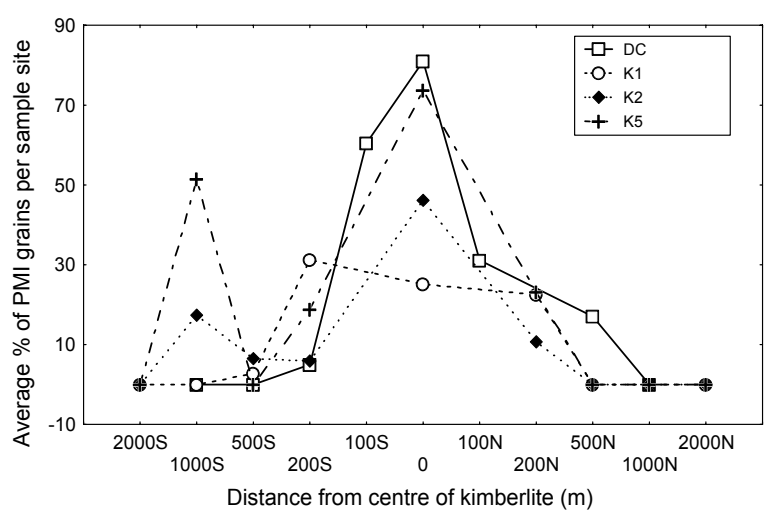

Figure 5: Decrease in Percentage of PMIs with distance from all four kimberlite bodies at Locality 2

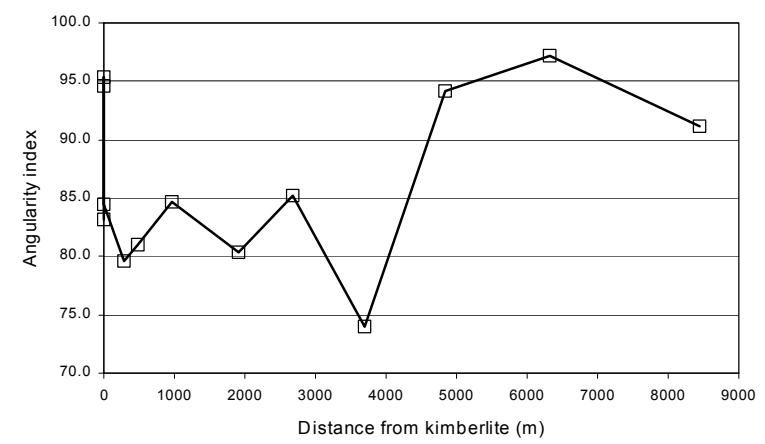

Figure 6: Increase in angularity of KIM grains, presented as an angularity index, relative to distance from the Locality 3 pipe. The grains were derived from deflation samples taken from within the dry river bed at Locality 3 .

\section{SAMPLE TECHNIQUES}

Five loam samples were collected directly over the Locality 1 kimberlite pipe. The KIM abundance recovered from each loam sample is, on average, of similar magnitude to that recovered from deflation samples collected at the same site (Table 2).

Loam and deflation sweep samples were collected at all Locality 2 sample sites. Figure 7 shows that a simple 1:1 relationship exists between the normalized KIM abundances recovered from deflation and loam samples collected at each site.
Table 2: A comparison of normalized KIM abundance recovered from deflation sweep and loam samples collected at 5 sites directly over the Locality 1 pipe.

\begin{tabular}{clc}
\hline \multirow{2}{*}{ Sample Site } & Sample Type & Normalized Total \\
\hline \hline \multirow{2}{*}{1} & Deflation & 131.3 \\
& Loam & 114.9 \\
\hline \multirow{2}{*}{2} & Deflation & 112.7 \\
& Loam & 134.2 \\
\hline \multirow{2}{*}{3} & Deflation & 119.5 \\
& Loam & 68.0 \\
\hline \multirow{2}{*}{4} & Deflation & 44.2 \\
& Loam & 110.0 \\
\hline \multirow{2}{*}{5} & Deflation & 141.3 \\
& Loam & 111.9 \\
\hline
\end{tabular}

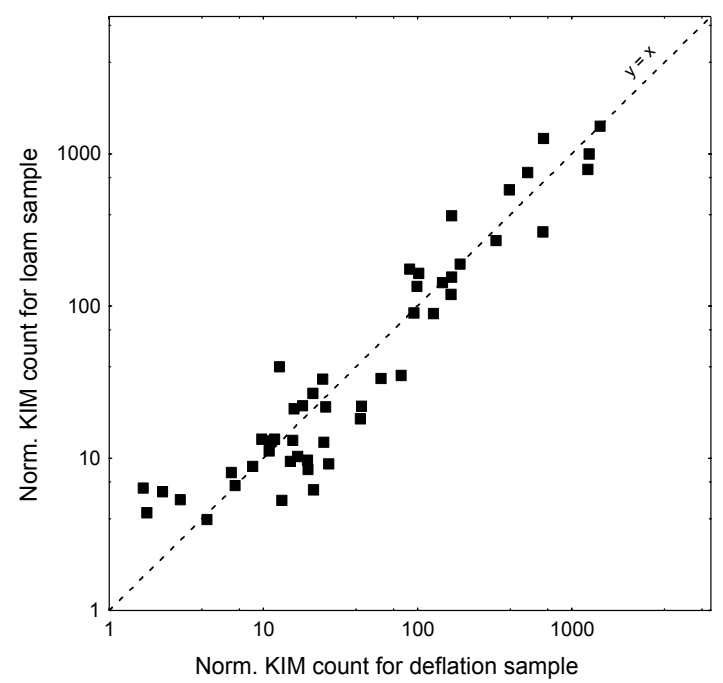

Figure 7: Simple 1:1 relationship observed for the abundance of KIMs recovered from loam and deflation samples collected at the same site at Locality 2

\section{HEAVy Mineral TRAP SITES}

Locality 1 and 2 show no consistent correlation between KIM abundance and trapsite quality for deflation sweep samples collected. However, a comparison of KIM abundance with the geomorphic feature recorded at each site indicates that anthills, deflation hollows and dry streams returned high KIM abundances.

The highest abundance of KIMs recovered at locality 3 were from deflation samples collected on the surface of 
medium to low energy gravel bars present within the dry river bed.

\section{CONCLUSIONS}

The following conclusions can be drawn from the work presented.

$>$ Due to the restricted KIM halos present over the Locality 1 and Locality 2 kimberlites optimal exploration sample spacing is of critical importance when defining a sampling program in sand covered terrains.

$>$ The size and extent of KIM dispersion halos is likely to reflect the KIM content of the kimberlite source.

$>$ The distribution of KIMs in the predominantly aeolian transport environment at localities 1 and 2 is more restricted than at Locality 3 where fluvial processes dominate.

$>$ KIMs recovered in exploration programs must be prioritized according to their size and surface texture classification.

$>$ There is no consistent enrichment of KIMs within deflation surfaces relative to underlying loam material, for samples taken over or proximal to kimberlite pipes in arid sand covered terrains.

$>$ The abundance of KIMs within deflation samples taken for this study does not correlate with trapsite quality. KIM abundance appears to be more directly related to geomorphological features present.

It is important to realize that factors such as the nature of sediment transport processes and the local geomorphology, physiography, depth of burial, vegetation cover and anticipated geology of the kimberlite target must be taken into account when attempting to apply these conclusions to other exploration projects

Contact: BR van Coller, PO Box 38668, Pinelands, Cape

Town, South Africa, 7430, E-mail:

brett.vancoller@minserv.co.za 